\title{
Cambios en la comunidad de peces por efecto del desarrollo costero en el Parque Nacional Huatulco (México)
}

Luis Gibran Juárez-Hernández ${ }^{1,2}$ * \& Margarito Tapia-García²

1. Centro Universitario CIFE. Calle Tabachin No. 514, Col. Bellavista. C. P. 62140, Cuernavaca, Morelos, México; gibbjuarez@gmail.com

2. Departamento de Hidrobiología. Universidad Autónoma Metropolitana. Unidad Iztapalapa. Av. San Rafael Atlixco No.186, Col. Vicentina. C.P. 09340. Ciudad de México, México; mtg@xanum.uam.mx

Recibido 22-V-2018. Corregido 08-VIII-2018. Aceptado 28-IX-2018.

\begin{abstract}
Changes in fish communities from the effect of coastal development in Huatulco National Park (Mexico). Coastal development and urbanization have increased the sediments that are washed from the land surface, producing adverse effects on the structure and functioning of coral reefs. The objective of this study was to identify the degree to which excessive sediments have disturbed the fish community in the coral reefs in the Maguey and Violin bays, located in the Huatulco National Park (Mexico). Fish samples were collected before and after disturbances produced by sediments associated with coastal development projects. Indicators of changes in the fish communities included the number and diversity of species as well as equity and dominance before and after the disturbances. The analysis was performed through means comparison tests, similarity analysis (ANOSIM) and similarity percentage analysis (SIMPER). In both locations, it was observed that the diversity, evenness, abundance and number of species were higher prior to the disturbance, but not for dominance, which shows an inverse pattern. In Maguey, significant differences in evenness and dominance were observed $(\mathrm{U}=28, \mathrm{p}=0.0401 ; \mathrm{U}=24, \mathrm{p}=0.0472$ ), as well as in species composition and abundance (ANOSIM $=0.35, \mathrm{p}$ $=0.009$ ). The similarity percentage analysis (SIMPER) indicated that after the disturbance, more than $46 \%$ of the species showed decreases in average abundance, highlighting the absence of Prionurus laticlavius, as well as the decrease in abundance of: P. punctatus, Cirrhitichthys oxycephalus, Microspathodon dorsalis, Lutjanus novemfaciatus and Stegates acapulcoensis. Inadequate planning and implementation of coastal development projects that contribute to the discharge of excessive sediments into the sea were determinants of negative changes in the coral communities in Maguey and Violin, whose effects could be seen in the composition of the species and the abundance, equity, and dominance of the fish community in Maguey.
\end{abstract}

Key words: ecological impact; environmental deterioration; Huatulco; ichthyofauna; coastal development; disturbance; community structure.

Juárez-Hernández, L. G., \& Tapia-García, M. (2018). Cambios en la comunidad de peces por efecto del desarrollo costero en el Parque Nacional Huatulco (México). Revista de Biología Tropical, 66(4), 1569-1579.

Los arrecifes coralinos son considerados como uno de los ecosistemas marinos de mayor vulnerabilidad, ya que durante las tres últimas décadas se ha observado la degradación y extensa mortalidad de corales pétreos, en áreas cercanas a desarrollos humanos, así como en arrecifes remotos y aislados (Hoegh-Guldberg,
1999; Burke, Reytar, Spalding, \& Perry, 2011). Las causas principales de deterioro y mortalidad se han clasificado como naturales (mareas bajas, ciclones, huracanes, tsunamis, variaciones severas de la temperatura oceánica) (Bruno \& Selig, 2007; Graham, Jennings, MacNeil, Mouillot, \& Wilson, 2015), y de origen 
antrópico las cuáles han sido consideradas como las de mayor influencia en la mortalidad y degradación de los arrecifes (Hughes et al., 2003; Mora, 2015). Esta relación se fundamenta por el creciente desarrollo costero y urbanización derivado del potencial económico que poseen estos ecosistemas (Hoegh-Guldberg, 1999; Cesar, Burke, \& Pet-Soede, 2003; Erftemeijer et al., 2012b; Rieg, Sheppard, \& Purkis, 2012).

El desarrollo costero en estos ecosistemas puede propiciar el ingreso de contaminantes, toxinas, e incremento en las tasas de sedimentación, provocando el decremento de la cobertura y riqueza coralina (Erftemeijer et al., 2012b), e influyendo en la composición del bentos arrecifal (Brodie et al., 2012; Halpern, Selkoe, White, Albert, Aswani, \& Lauer, 2013). A este respecto, los arrecifes coralinos son particularmente sensibles a incrementos en las tasas de sedimentación, ya que se altera la composición y estructura de la comunidad, así como se modifica la frecuencia de tallas, tamaño de las colonias coralinas, y minimiza las tasas de supervivencia y establecimiento (Piniak \& Brown, 2008; Erftemeijer, Hagedorn, Laterveer, Craggs, \& Guest, 2012a), propiciando cambios en otros miembros de la fauna arrecifal, incluidos los peces (Rogers, 1990; Syms \& Jones, 2000; Wenger, Johansen, \& Jones, 2011). Los efectos de estas perturbaciones (i.e. sedimentación) en la comunidad íctica se han relacionado con cambios en su abundancia y diversidad (Lewis, 1997; Halford, Cheal, Ryan, \& Williams, 2004; Jones, McCormick, Srinivasan, \& Eagle, 2004; Holdbrook, Brooks, \& Schmitt, 2006; Rogers, Blanchard, \& Mumby, 2014), decremento en los procesos de colonización y reclutamiento (Lewis, 1998), debido a la reducción de la actividad reproductiva y condición fisiológica (Syms \& Jones, 2000; Munday, 2004).

Las comunidades coralinas de las bahías de Huatulco (México) han sufrido alteraciones por factores naturales (Lirman, Glynn, Baker, \& Leyte Morales, 2001; López-Pérez, Hernández-Ballesteros, \& Herrera-Escalante, 2002; López-Pérez et al., 2016) y antropogénicos
(Glynn \& Leyte-Morales, 1997). En relación con las alteraciones antropogénicas, se ha señalado que el proceso de urbanización del área en su primera etapa implicó la apertura de áreas de selva, la remoción de suelos y la modificación de afluentes naturales, implicando la alteración y en algunos casos la pérdida de las comunidades coralinas (López-Pérez et al., 2002; CONANP, 2003; Granja-Fernández \& López-Pérez, 2008). En la última década, por su potencial turístico, la zona ha sido sujeta a una creciente presión determinada por la urbanización, desarrollo de la infraestructura turística y actividades turísticas, manifestándose en problemas de calidad ambiental, contaminación y degradación (Magaña-Melgoza \& VegaGonzález, 1991; López-Pérez et al., 2002; López-Pérez \& Hernández-Ballesteros, 2004). Particularmente han destacado los relacionados con la construcción de andadores escénicos por parte del Fondo Nacional de Fomento al Turismo (FONATUR), generando perturbación al ambiente por el ingreso de sedimento terrígeno en las bahías de Maguey y Violín (Castañeda, 2009; Vélez-Ascencio, 2009; Mendoza, 2011; PROFEPA, 2011).

Para el área de estudio, no existen antecedentes de la influencia de los factores asociados al desarrollo costero en la ictiofauna, por lo tanto y considerando la importancia de conocer el efecto del aporte de sedimentos por obras de desarrollo hacia estos ecosistemas, el objetivo del estudio fue determinar los cambios en la estructura de las comunidades de peces de las bahías Maguey y Violín antes y después de la perturbación.

\section{MATERIALES Y MÉTODOS}

El Parque Nacional Huatulco (PNH), se localiza en la costa de Oaxaca entre los para-

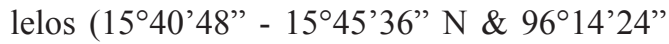
- 96 $06^{\circ} 13^{\prime \prime}$ W) (Fig. 1), y agrupa las bahías de San Agustín, Riscalillo, Chachacual, Cacalutla, Maguey, Órgano y Violín. En el área, el ciclo anual se caracteriza por la época de secas (noviembre-abril) y lluvias (mayo-octubre). Respecto a las tasas de sedimentación, de 
manera natural se registra un mayor aporte a la comunidad coralina en época de lluvias (74.5-147.6 $\mathrm{mg} \mathrm{cm}^{-2}$ día $\left.^{-1}\right)$ por el incremento de escorrentías asociadas, destacando que los valores en las tasas de sedimentación podrían considerarse potencialmente crónicos; sin embargo, el efecto puede ser considerado como reducido resultado del nivel de desarrollo coralino (Glynn \& Leyte-Morales 1997; Granja-Fernández \& López-Pérez, 2008). La temperatura promedio del agua superficial es de 26 a $28^{\circ} \mathrm{C}$ (Fiedler, 1992), con un promedio anual de $25^{\circ} \mathrm{C}$ (Tapia-García, García-Abad, CarranzaEdwards, \& Vázquez-Gutiérrez, 2007).

La zona es relevante ya que alberga una riqueza considerable de especies de equinodermos (32 sp.), corales pétreos (10 sp.) y peces (150-165 sp.) respecto a otras áreas naturales protegidas en el Pacífico central mexicano (López-Pérez et al., 2014; Juárez-Hernández \& Tapia-García, 2017; 2018). Respecto a la ictiofauna, temporalmente se ha indicado que la mayor riqueza de especies y diversidad de peces se presentan en la época de lluvias (López-Pérez, Calderón-Aguilera, ZepedaVilchis, López Pérez-Maldonado, \& LópezOrtiz, 2012; Juárez-Hernández, Tapia-García, \& Luna-Monsiváis 2013; Juárez-Hernández, 2014). Dentro de las bahías, Maguey y Violín (Fig. 1), han sido consideradas como de las de mayor riqueza íctica (69 y $50 \mathrm{sp}$.) y coralina (8 y 9 sp.) (López-Pérez et al., 2012; JuárezHernández \& Tapia-García, 2017).

Durante la caracterización de las comunidades ícticas de Bahías de Huatulco (JuárezHernández \& Tapia-García, 2017), dos eventos de perturbación se presentaron en la Bahía Maguey y Violín en enero 2009 y julio 2010 respectivamente. Como se mencionó, estos eventos fueron propiciados por el ingreso de sedimento terrígeno resultado de la construcción de andadores escénicos. Particularmente el daño sobre la comunidad coralina en Maguey fue observable en los transectos siete, ocho y nueve, los cuales son de tipo coralino (Fig. 1), y en Bahía Violín el daño en la comunidad coralina fue en los transectos cinco, seis y siete (Fig. 1), los cuales se caracterizaron por presentar una mayor representación de coral vivo (Juárez-Hernández, 2014) (Fig. 1). Para determinar el efecto de estos eventos en la comunidad íctica, se caracterizó la comunidad de peces a través de la técnica de censos visuales, mediante transectos de $10 \mathrm{~m}$ de longitud y $2.5 \mathrm{~m}$ de ancho entre enero 2009 y diciembre 2010. Específicamente en Maguey se realizaron 24 transectos (10 transectos antes de la perturbación y 14 transectos posterior a la perturbación) y en Violín se realizaron 17 transectos (nueve antes de la perturbación y ocho posterior). Para la comunidad coralina se realizaron observaciones de la mortalidad coralina y crecimiento algal en un muestreo posterior a la perturbación, de acuerdo con lo propuesto por Raymundo, Couch, \& Harvell (2008) así como Lasagna et al. (2014).

Para la identificación de las especies ícticas se consultaron los trabajos de Allen \& Robertson (1998) y Amezcua-Linares (2009). Posteriormente se determinó el número de especies, abundancia, diversidad $\left(\mathrm{H}^{\prime}\right)$ (bits/ individuo) (Shannon \& Wiener, 1963), equidad J' (Pielou, 1977) y dominancia (D') (Simpson, 1968), de la comunidad íctica previo y posterior de los eventos de perturbación. El análisis de estos atributos se realizó mediante pruebas de $\mathrm{t}$, cuando se cumplió el supuesto de normalidad (prueba de Shapiro-Wilk, $p>0.05$ ) y homocedasticidad (prueba de Levene, $\mathrm{p}>0.05$ ). Si los supuestos no eran comprobables, se aplicó la prueba U de Mann-Whitney. Se realizó un análisis de similitud (ANOSIM) (Clarke, 1993) para la identificación de diferencias significativas en términos de composición y abundancia íctica. Finalmente, si el análisis de similitud denotaba diferencias significativas, se realizó un análisis de porcentaje de similitud (SIMPER) con el propósito de identificar las especies responsables de la diferenciación. Estos análisis se realizaron empleando la matriz de similitud mediante el índice de BrayCurtis, con el paquete PRIMER v5 (Clarke \& Gorley, 2006).

Figuras y cuadros: las figuras y cuadros se encuentran en el Apéndice Digital 1. 


\section{RESULTADOS}

En enero 2009 en Bahía Maguey se observó turbidez, así como la presencia de mucus sobre los corales, posteriormente en marzo del mismo año se determinó una mortalidad coralina superior del $95 \%$, observándose crecimiento masivo de algas calcáreas sobre las colonias coralinas en los transectos señalados (Fig. 2B y 2C). Para Bahía Violín, en los transectos analizados, se observó en noviembre 2009 mortalidad del $80 \%$ y un crecimiento de algas calcáreas (Fig. 3C y 3D). Para ambas localidades, se destaca que el resto de los ambientes no presentaron signos evidentes de alteración (i. e. mucus, mortalidad coralina, crecimiento algal).

En ambas localidades la diversidad, equidad, abundancia y número de especies de peces fue mayor antes del evento de perturbación, no siendo así para la dominancia, la cual exhibió un patrón inverso (Cuadro 1). En Maguey se observó un decremento del $26 \%$ de la abundancia total, mientras que en Violín el decremento fue superior al $28 \%$. Respecto a la diversidad, en ambas localidades se presentó un decremento, siendo mayor en Bahía Maguey (Cuadro 1). El análisis de estos atributos demostró que únicamente la equidad $(\mathrm{U}=28, \mathrm{p}=0.0401) \mathrm{y}$ dominancia $(\mathrm{U}=24, \mathrm{p}=0.0472$ ) fueron significativamente diferentes antes y después de la perturbación en Bahía Maguey. El análisis de similitud indicó que la composición y abundancia íctica presentó diferencias antes y después de la mortalidad coralina $(\mathrm{R}=0.35, \mathrm{p}=0.009)$ en Maguey. En Bahía Violín, el análisis de similitud no reveló diferenciación $(\mathrm{R}=0.1, \mathrm{p}>0.05)$. En Maguey el análisis de similitud porcentual (SIMPER), indicó que la disimilitud promedio antes y después del disturbio fue de más del $51 \%$. A este respecto, el análisis señaló que más de 26 especies explicaron el $90 \%$ de la diferenciación. Este análisis reveló que Prionurus laticlavius no fue observada posterior a la perturbación, mientras que: $P$. punctatus, Cirrhitichthys oxycephalus, Microspathodon bairdii, Stegastes flavilatus, Lutjanus argentiventris, Epinephelus labriformes, Holacanthus passer, Johnrandallia nigrirostris, M. dorsalis,
L. novemfaciatus y S. acapulcoensis, presentaron decrementos en su abundancia promedio. Contrariamente, Thalassoma lucasanum, Halichoeres dispilus, Bodianus diplotaenia, $H$. notospilus, Ophioblennius steindachneri, Cephalophis panamensis, H, chierchiae, Caranx caballus, Fistularia comersonii, Scarus compressus, $S$. rectifraenum, $K$. analogus y $H$. nicholsi mostraron incrementos en su abundancia promedio (Cuadro 2).

\section{DISCUSIÓN}

Para el área de estudio se han reportado eventos que han alterado la comunidad coralina, los cuales se relacionan con cambios en la temperatura del mar (López-Pérez et al., 2002; 2016). Para el presente estudio se señala que la alteración observada en las comunidades coralinas fue determinada por aportes puntuales de sedimento terrígeno derivado de la construcción de los andares escénicos. A este respecto, el evento de perturbación sobre la comunidad coralina en Maguey se relacionó con la construcción de un andador escénico por parte FONATUR, el cual se inició a finales del 2008 y contemplaba la comunicación con Punta Maguey (Castañeda, 2009; VélezAscencio, 2009). Esta obra fue clausurada por la Procuraduría Federal de Protección Ambiental (PROFEPA), resultado de la alteración de áreas destinadas a la conservación y se emitió una sanción económica a esta institución (FONATUR). En Violín, resultado de la construcción del andador escénico "Sección de Andador de Usos Múltiples Escénico II" por parte de FONATUR en el 2010, la inadecuada disposición del material terrígeno derivado de la construcción de este andador fue arrastrado hacia el interior de la bahía en julio 2010, por efecto de las lluvias (Fig. 3A y 3B). Esta obra fue clausurada por parte de PROFEPA e impuso una sanción económica a los desarrolladores de esta obra (Mendoza, 2011; PROFEPA, 2011). En este sentido, es importante señalar que para el resto de transectos, en lo que el sustrato de mayor representación es el coralino no se observó mortalidad coralina o cambios 
de fase (coral-algal), lo anterior denotaría la ausencia de factores de orden generalizado como cambios en la temperatura del mar. Otro aspecto relevante observado, y que apoyaría la naturaleza de la perturbación, fue la presencia de mucus en los ambientes impactados, lo cual de acuerdo con Erftemeijer et al. (2012a), se considera un mecanismo para hacer frente a las elevadas tasas de sedimentación. Respecto al cambio de fase observado, se indica que los eventos de perturbación en la comunidad coralina minimizan su capacidad competitiva frente a otros organismos, como las macroalgas, ya que la disponibilidad de espacio posterior a la alteración permite la colonización de un ensamblaje diverso de algas filamentosas y calcáreas (Diaz-Pulido \& McCook, 2004; Enochs \& Hockensmith, 2008).

Los eventos de perturbación que involucren mortalidad coralina determinan cambios en la composición y estructura de la comunidad íctica (Rogers, 1990; Lewis, 1997; Syms \& Jones, 2000; Wenger, Johansen, \& Jones, 2011). Lo anterior es consistente con los resultados del presente estudio, particularmente en lo referente a Maguey en donde se presentaron diferencias significativas en la composición, abundancia, equidad y dominancia de la comunidad íctica, antes y después de la perturbación. Diversos autores concluyen que los cambios en la ictiofauna son resultado de la reducción en la variedad y calidad de hábitats, así como los recursos disponibles a macro y microescala por la pérdida de la complejidad y estructura del hábitat (Halford et al., 2004; Jones et al., 2004; Munday, 2004; Garpe, Yahya, Lindahl, \& Öhman, 2006; Graham, Wilson, Jennings, Polunin, Bijoux, \& Robinson, 2006; Holbrook, Brooks, \& Schmitt, 2006; Wilson et al., 2006; Erftemeijer et al., 2012b; Rogers et al., 2014). Es importante señalar que para el área de estudio se han reportado decrementos en la abundancia y diversidad relacionados con la alteración de la comunidad coralina por factores antrópicos y naturales, en este orden Ramírez-Gutiérrez (2010) indica que los impactos acumulados por actividades turísticas acuáticas (buceo libre) sobre la comunidad coralina de
Bahía San Agustín determino el decremento en la diversidad y abundancia de la ictiofauna. Por su parte López-Pérez et al. (2016) refieren cambios en la abundancia de diversas especies de peces resultado de decrementos en la cobertura de coral por incremento en la temperatura del mar.

Diversos autores mencionan que posterior a un evento de mortalidad coralina, aproximadamente más del $30 \%$ especies de peces exhiben cambios significativos en su abundancia con un rango de respuesta desde extinciones locales hasta decrementos e incrementos en la abundancia (Booth \& Beretta, 2002; Spalding \& Jarvis, 2002; Munday, 2004). Correspondiendo con lo anterior, el análisis empleado reveló que más del $46 \%$ de especies en Maguey presentaron decrementos en la abundancia promedio posterior a la perturbación, destacando la ausencia de $P$. laticlavius, así como el decremento en la abundancia de 11 especies. Dentro de este conjunto, se refieren especies que han sido consideradas como dominantes para el área de estudio (i. e. $M$. dorsalis y $S$. acapulcoensis), así como especies que poseen una escasa representación numérica y espacial (C. oxycephalus, P. laticlavius y L. novemfasciatus) (López-Pérez et al., 2010; 2012; JuárezHernández \& Tapia-García, 2017; 2018). De las especies que incrementaron su abundancia posterior a la perturbación se destaca a $T$. lucasamun, la cual es considerada como especie dominante (Juárez-Hernández \& Tapia-García, 2017), así como especies que son abundantes (O. steindachneri, B. diplotaenia, H. dispilus, H. notospilus, C. caballus) (López-Pérez et al., 2010, Juárez-Hernández \& Tapia-García, 2017)

Comeros-Raynal et al. (2012) refieren que los miembros de la familia Acanthuridae presentan una fuerte asociación con los arrecifes coralinos, por lo tanto, la pérdida de cobertura coralina puede determinar extinciones locales, así como el decremento en su abundancia. Lo anterior es consistente con los resultados del presente, ya que como se indicó P. laticlavius no fue observada posterior al evento de perturbación, mientras que para $P$. punctatus decrece su abundancia. El efecto diferencial 
en estas especies, probablemente se explique por la mayor especificidad en el espectro alimenticio de P. laticlavius (Moreno-Sánchez, 2009). Respecto al decremento en la abundancia de C. oxycephalus se podría explicar por la dependencia directa con los corales, ya que vive, se alimenta y reproduce dentro de la colonia coralina (Thomson, Findley, \& Kerstitch, 2000), presentando una mayor afinidad a áreas de gran cobertura coralina y de alta complejidad (González-Cabello \& Bellwood, 2009; Moreno-Sánchez, 2009), por lo anterior posee una mayor sensibilidad a la alteración en las comunidades coralinas que involucren la perdida de coral vivo (Halford et al., 2004; Jones et al., 2004; Pratchett, Wilson, \& Baird, 2006; Wilson et al., 2006).

Lewis (1998) indica que los eventos de perturbación en arrecifes coralinos determinan la reducción de la biomasa criptica (invertebrados). En este sentido, los invertebrados es un componente que representa una parte integral en la dinámica de nutrientes y redes tróficas, siendo la fuente alimenticia para un gran número de familias de peces como Balistidae, Cirrhitidae, Haemuldae, Lutjanidae, Labridae y Serranidae (Enochs \& Hockensmith; 2008). Concordando con estos argumentos, probablemente el decremento en la abundancia de $L$. argentiventris así como $H$. passer se relacionan con la pérdida de recursos alimenticios y sitios de refugio (Pérez-España \& Abitia-Cárdenas, 1996; Robertson \& Allen, 2015; López-Pérez et al., 2012). Otra de las especies que mostró decrementos en su abundancia fue $J$. nigrirostris, la cual se ha clasificado como una especie coralívora facultativa (Allen \& Robertson, 1998; Robertson \& Allen, 2015). Las especies pertenecientes a este grupo son afectadas en menor medida en decrementos moderados de cobertura coralina, incrementando la ingesta de presas no coralinas, sin embargo, los corales representan un componente necesario e importante en su dieta, por lo que aun estas especies son afectadas por la pérdida de coral vivo (Pratchett et al., 2006; 2008; Wilson et al., 2006).

Para M. dorsalis y M. bairdii, como especies herbívoras se esperaría un incremento en su abundancia, presumiblemente debido a que un mayor número de áreas comienzan a ser disponibles para el crecimiento algal posterior a la perdida de coral (Wilson et al., 2006). Sin embargo, este grupo sufre decrementos en su abundancia, relacionada con la perdida de la estructura y complejidad del hábitat (Garpe et al., 2006), ejerciendo un mayor efecto en los miembros de familia Pomacentridae (Lewis, 1998). Este argumento coincide con los decrementos en la abundancia de ambas especies ya que se distribuyen en áreas topográficamente complejas (López-Pérez et al., 2012; Juárez-Hernández, 2008). Además, el cambio de fase (coral a algal) determinaría el colapso de las especies algales de las que regularmente se alimentan estas damiselas herbívoras (Dominici-Arosemena \& Wolff, 2006). Probablemente este cambio de fase también ejerce influencia en el decremento en la abundancia de otras especies pertenecientes a esta familia (i. e. Stegastes flavilatus y S. acapulcoensis) y grupo trófico.

Syms \& Jones (2000), así como Glynn (2006), señalan que las especies que probablemente se beneficien de la perdida coralina y degradación arrecifal, son las de tallas pequeñas, generalistas y herbívoros que habitan áreas de bajo relieve y escombro, como algunas especies de labridos, blenidos y algunos pomacéntridos, lo anterior coincide con el incremento en la abundancia de las especies pertenecientes al género Halichoeres, así como para: T. lucasanum, S. rectifraenum, S. compressus y $O$. steindachneri, las cuales presentan su mayor abundancia en áreas de escaso relieve como escombro coralino, coral muerto y ambientes con una dominancia del sustrato arenoso (Dominici-Arosemena \& Wolff, 2006; López-Pérez et al., 2012; Juárez-Hernández \& Tapia-García, 2017).

Granja-Fernández \& López-Pérez (2008) señalan que la ausencia de mortalidad coralina en respuesta a los niveles de sedimentación registrados durante la época de lluvias, y el desarrollo coralino observado en la región pudiera estar relacionado al efecto combinado de la eficiencia de la remoción activa de 
sedimento, así como el posible incremento en la tolerancia fisiológica al sedimento por parte de los corales pétreos de Bahías de Huatulco. A este respecto, en una visita prospectiva realizada a mediados del 2011 se observaron signos de recuperación de la comunidad coralina en el área impacta de Violín, con una reducción en la cobertura algal, así como la presencia de nuevos reclutas coralinos. Probablemente la mayor resiliencia en esta localidad se relacione con el intenso oleaje y ausencia de agentes de estrés antropogénico. En este sentido, Riegl, Heine, \& Branch (1996), Hubmann, Piller, \& Riegl (2002) así como Shedrawi et al. (2017), señalan que los corales que se suscriben y crecen en áreas de oleaje moderado a intenso, generalmente no requieren de mecanismos activos (i. e. producción de mucus) de eliminación de sedimentos, ya que la turbulencia generada por el oleaje asiste en la eliminación pasiva de sedimento acumulado en el tejido coralino. Aunado a este hecho se indica que esta localidad no posee infraestructura turística y su acceso es limitado, por lo que la presencia de turistas es escasa. Este aspecto es relevante ya que la ausencia de agentes de estrés de origen antropogénico promueve la resiliencia en las comunidades coralinas impactadas (West \& Salm, 2003). Contrariamente en Maguey, no se registraron indicios de recuperación, observándose una progresiva y amplia degradación de la comunidad coralina en los ambientes impactados, la cual puede estar influida principalmente por frecuencia agentes de estrés determinados por la infraestructura turística que posee, y probablemente por el nulo oleaje imperante en esta localidad.

La inadecuada planeación y ejecución de proyectos de desarrollo costero determinó el ingreso de sedimento propiciando la alteración de las comunidades coralinas de Maguey y Violín (en algunos casos el daño total y perpetuo), resultando en cambios significativos en la composición, abundancia, equidad y dominancia de la ictiofauna de Maguey. Aunado a lo anterior, la escasa regulación de actividades turísticas acuáticas añade impactos negativos a la comunidad coralina e íctica
(Ramírez-Gutiérrez，2010; Juárez-Hernández \& Tapia-García, 2017). Ante el incremento en la urbanización y el desarrollo de infraestructura turística se sugiere una revisión extensiva de los planes de manejo, así como la actuación sinérgica de las autoridades competentes, que permita conservar y preservar el valor biológico, ecológico y económico de las comunidades coralinas del Parque Nacional Huatulco.

Declaración de ética: los autores declaran que todos están de acuerdo con esta publicación y que han hecho aportes que justifican su autoría; que no hay conflicto de interés de cualquier tipo; y que han cumplido con todos los requisitos y procedimientos éticos y legales pertinentes. El documento firmado se encuentra en los archivos de la revista.

\section{AGRADECIMIENTOS}

Este trabajo se realizó a través del proyecto de investigación "Diagnostico ecológico de los sistemas acuáticos de México, como base para su gestión ambiental" del departamento de Hidrobiología de la Universidad Autónoma Metropolitana, Unidad Iztapalapa. A las autoridades y personal del Parque Nacional Huatulco, por el apoyo y disposición para la autorización y ejecución del presente estudio.

\section{RESUMEN}

El incremento del aporte de sedimentos desde tierra derivado del desarrollo costero y urbanización ha producido efectos adversos en la estructura y función de los arrecifes coralinos. El objetivo de este estudio fue conocer la magnitud de disturbios por aportes excesivos de sedimentos en la comunidad íctica de arrecifes coralinos de las bahías Maguey y Violín del Parque Nacional Huatulco (México). Los muestreos de peces se realizaron antes y después de disturbios ocasionados por aportes de sedimentos asociados a obras de desarrollo costero. Como indicadores de cambios en las comunidades de peces se utilizaron el número de especies, diversidad, equidad y dominancia antes y después de la perturbación. El análisis se realizó mediante pruebas de comparación de medias, análisis de similitud (ANOSIM) y análisis de porcentaje de similitud (SIMPER). En ambas localidades, se observó, que, excepto la dominancia, la diversidad, equidad, 
abundancia y número de especies fueron mayores previos a la perturbación. En Maguey se observaron diferencias significativas en la equidad y dominancia $(\mathrm{U}=28, \mathrm{p}=0.0401$; $\mathrm{U}=24, \mathrm{p}=0.0472$ ), así como en la composición de especies y la abundancia (ANOSIM=0.35, p=0.009). El análisis de porcentaje de similitud (SIMPER) indicó que posterior a la perturbación, el $46 \%$ de especies presentaron una disminución en su abundancia, destacó la ausencia de Prionurus laticlavius, así como el decremento en la abundancia de: P. punctatus, Cirrhitichthys oxycephalus, Microspathodon dorsalis, Lutjanus novemfaciatus y Stegates acapulcoensis. La inadecuada planeación y ejecución de proyectos de desarrollo costero que propiciaron aporte excesivo de sedimentos al mar determinaron cambios negativos en las comunidades coralinas de Maguey y Violín, cuyo efecto fue observado en la composición de especies, abundancia, equidad y dominancia de la comunidad de peces de Maguey.

Palabras clave: impacto ecológico; deterioro ambiental; Huatulco; ictiofauna; desarrollo costero; perturbación; estructura de la comunidad.

\section{REFERENCIAS}

Allen, G. R., \& Robertson, D. R. (1998). Peces del Pacífico Oriental Tropical. México: CONABIO, agrupación Sierra Madre y CEMEX.

Amezcua-Linares, F. (2009). Peces demersales del Pacifico de México. México: Instituto de Ciencias Marinas y Limnología, Universidad Nacional Autónoma de México.

Booth, D. J., \& Beretta, G. A. (2002). Changes in a fish assemblage after a coral bleaching event. Marine Ecology Progress Series, 245, 205-212.

Brodie, J. E., Kroon, F. J., Schaffelke, B., Wolanski, E. C., Lewis, S. E., Devlin, M. J., ... \& Davis, A. M. (2012). Terrestrial pollutant runoff to the Great Barrier Reef: an update of issues, priorities and management responses. Marine Pollution Bulletin, 65(4), 81-100.

Bruno, J. F., \& Selig, E. R. (2007). Regional decline of coral cover in the Indo-Pacific: timing, extent, and subregional comparisons. PLOS ONE, 2(8), e711. doi: 10.1371/journal.pone.0000711

Burke, L., Reytar, K., Spalding, M., \& Perry, A. (2011). Reefs at Risk revisited. Washington D. C.: World Res Institute.

Castañeda, J. (2009). Clausuró la Profepa dos obras de Fonatur en Huatulco. Notihuatulco Recuperado de http://huatulconoticias.com/?p=3174

Cesar, H., Burke, L., \& Pet-Soede, L. (2003). The economics of worldwide coral reef degradation.
Netherlands, Arnhem: Cesar Environmental Economics Consulting (CEEC).

Clarke, K. R. (1993). Non-parametric multivariate analysis of changes in community structure. Australian Journal of Ecology, 18, 117-143.

Clarke, K. R., \& Gorley, R. (2006). PRIMER V5: User Manual/ Tutorial. Plymouth, UK.: PRIMER-E. Recuperado de www.primer-e.com

Comisión Nacional de Áreas Naturales Protegidas (CONANP). (2003). Programa de manejo Parque Nacional Huatulco. México, D. F.: Dirección General de Manejo para la Conservación, Comisión Nacional de Áreas Naturales Protegidas.

Comeros-Raynal, M. T., Choat, J. H., Polidoro, B. A., Clements, K. D., Abesamis, R., Craig, M.T., ... Nañola Jr., C. L. (2012). The likelihood of extinction of iconic and dominant herbivores and detritivores of coral reefs: the parrotfishes and surgeonfishes. PLOS ONE 7(7), e39825. doi: 10.1371/journal.pone.0039825

Diaz-Pulido, G., \& McCook, L. J. (2004). Effects of live coral, epilithic algal communities and substrate type on algal recruitment. Coral Reefs, 23(2), 225-233.

Dominici-Arosemena, A., \& Wolff, M. (2006). Reef fish community structure in the Tropical Eastern Pacific (Panamá): living on a relatively stable rocky reef environment. Helgoland Marine Research, 60(4), 287-305.

Enochs, I. C., \& Hockensmith, G. (2008, Julio). Effects of coral mortality on the community composition of cryptic metazoans associated with Pocillopora damicornis. En A. Kerr, G. Paulay, \& M. Picho (Chairs), Biodiversity and Diversification of Reef Organisms. 11th International Coral Reef Symposium Conference, Fort Lauderdale, Florida.

Erftemeijer, P. L., Hagedorn, M., Laterveer, M., Craggs, J., \& Guest, J. R. (2012a). Effect of suspended sediment on fertilization success in the scleractinian coral Pectinia lactuca. Journal of the Marine Biological Association of the United Kingdom, 92(04), 741-745.

Erftemeijer, P. L., Rieglc, B., Hoeksemad, B. W., \& Todde, P. A. (2012b). Environmental impacts of dredging and other sediment disturbances on corals: a review. Marine Pollution Bulletin, 64, 1737-1765.

Fiedler, P. C. (1992). Seasonal climatologies and variability of Eastern Tropical Pacific Surface waters. NOAA Technical reports NMFS, 109, 1-65.

Garpe, K. C., Yahya, S. A., Lindahl, U., \& Öhman, M. C. (2006). Long-term effects of the 1998 coral bleaching event on reef fish assemblages. Marine Ecology Progress Series, 315, 237-247.

Glynn, P. W. (2006, Junio). Fish utilization of simulated coral reef frameworks versus eroded rubble 
substrates off Panama, eastern Pacific. Sesión de presentaciones orales Changing coral communities: What are the consequences for coral reef fishes? 10th International Coral Reef Symposium Conference, Okinawa, Japón.

Glynn, P. W., \& Leyte Morales, G. E. (1997). Coral reefs of Huatulco, West México. Revista de Biología Tropical, 45(3), 1033-1047.

González-Cabello, A., \& Bellwood, D. R. (2009). Local ecological impacts of regional biodiversity on reef fish assemblages. Journal of Biogeography, 36(6), $1129-1137$

Graham, N. A., Wilson, S. K., Jennings, S., Polunin, N. V., Bijoux, J. P., \& Robinson, J. (2006). Dynamic fragility of oceanic coral reef ecosystems. Proceedings of the National Academy of Sciences, 103(22), 8425-8429.

Graham, N. A., Jennings, S., MacNeil, M., Mouillot, D., \& Wilson, S. (2015). Predicting climate-driven regime shifts versus rebound potential in coral reefs. Nature, 518, 94-97.

Granja-Fernández, M. R., \& López-Pérez, R. A. (2008). Sedimentación en comunidades arrecifales de Bahías de Huatulco, Oaxaca, México. Revista de Biología Tropical, 56(3), 1179-1187.

Halford, A., Cheal, A. J., Ryan, D., \& Williams, D. M. (2004). Resilience to large-scale disturbance in coral and fish assemblages on the Great Barrier Reef, Australia. Ecology, 85(7), 1892-1905.

Halpern, B. S., Selkoe, K. A., White, C., Albert, S., Aswani, S., \& Lauer, M. (2013). Marine protected areas and resilience to sedimentation in the Solomon Islands. Coral Reefs, 32(1), 61-69.

Hoegh-Guldberg, O. (1999). Climate change, coral bleaching and the future of the world's coral reefs. Marine and Freshwater Research, 50(8), 839-866.

Holbrook, S. J., Brooks, A. J., \& Schmitt, R. J. (2006, Junio). Relationships between live coral cover and reef fishes: implications for predicting effects of environmental disturbances. Sesión de presentaciones orales. Changing coral communities: What are the consequences for coral reef fishes? 10th International Coral Reef Symposium Conference, Okinawa, Japón.

Hubmann, B., Piller, W. E., \& Riegl, B. (2002). Functional morphology of coral shape and passive hydrodynamic self-righting in recent Manicina areolata. Palaeobiodiversity and Palaeoenvironments, 82(1), 125-130.

Hughes, T. P., Baird, A. H., Bellwood, D. R., Card, M., Connolly, S. R., Folke, C., ... Lough, J. M. (2003). Climate change, human impacts, and the resilience of coral reefs. Science, 301(5635), 929-933.
Jones, G. P., McCormick, M. I., Srinivasan, M., \& Eagle, J. V. (2004). Coral decline threatens fish biodiversity in marine reserves. Proceedings of the National Academy of Sciences of the United States of America, 101(21), 8251-8253.

Juárez-Hernández, L. G. (2008). Composición, distribución y abundancia de la ictiofauna en la Bahía Maguey, Huatulco, Oaxaca, y su relación con la complejidad (estructura) del hábitat (Tesis de maestría). Universidad Autónoma Metropolitana-Iztapalapa, México.

Juárez-Hernández, L. G. (2014). Estructura de las comunidades de peces de las bahías del Parque Nacional Huatulco, con énfasis en los sistemas de arrecifes de coral (Tesis doctoral). Universidad Autónoma Metropolitana-Iztapalapa, México.

Juárez-Hernández，L. G., \& Tapia-García， M. (2017). Variación espacial en número de especies, abundancia y diversidad de peces en las Bahías de Huatulco, Oaxaca, México. Revista de Biología Tropical, 65(4), 1407-1418.

Juárez-Hernández, L. G., \& Tapia-García, M. (2018). Listado ictiofaunístico de las bahías del Parque Nacional Huatulco, Oaxaca, México. Arxius de Miscel·lània Zoològica, 16, 96-111.

Juárez-Hernández, L. G., Tapia-García, M., \& LunaMonsiváis, B. (2013). Estructura de las comunidades de peces de las bahías Maguey y Cacaluta, Huatulco, Oaxaca. Revista Mexicana de Biodiversidad, 84(4), 1243-1257.

Lasagna, R., Gnone, G., Taruffi, M., Morri, C., Bianchi, C. N., Parravicini, V., \& Lavorano, S. (2014). A new synthetic index to evaluate reef coral condition. Ecological Indicators, 40, 1-9.

Lewis, A. R. (1997). Effects of experimental coral disturbance on the structure of fish communities on large patch reefs. Marine Ecology Progress Series, 161, 37-50.

Lewis, A. R. (1998). Effects of experimental coral disturbance on the population dynamics of fishes on large patch reefs. Journal of Experimental Marine Biology and Ecology, 230(1), 91-110.

Lirman, D., Glynn, P. W., Baker, A. C., \& Leyte Morales, G. E. (2001). Combined effects of three sequential storms on the Huatulco coral reef tract, Mexico. Bulletin of Marine Science, 69(1), 267-278.

López-Pérez, R. A., Guendulain-García, S., Granja-Fernández, R., Hernández-Urraca, V., Galván-Rowland, L., Zepeta-Vilchis, R., \& López-López, D. (2016). Reef Community Changes Associated with the 2009-2010 El Niño in the Southern Mexican Pacific. Pacific Science, 70(2), 175-190.

López-Pérez, R. A., Hernández-Ballesteros, L. M., \& Herrera-Escalante, T. (2002). Cambio en la dominancia de 
la comunidad arrecifal en Chachacual, Bahías de Huatulco, Oaxaca. Ciencia y Mar, 16(4), 33-38.

López-Pérez, R. A., Calderón-Aguilera, L. E., ZepedaVilchis, R. C., López Pérez-Maldonado, I., \& LópezOrtiz, A. M. (2012). Species composition, habitat configuration and seasonal changes of coral reef fish assemblages in western Mexico. Journal of Applied Ichthyology, 29(2), 437-448.

López-Pérez, R. A., López Pérez-Maldonado, I., LópezOrtiz, A. M., Barranco-Servín, L. M., BarrientosVillalobos, J., \& Leyte-Morales, G. E. (2010). Reef fishes of the Mazunte-Bahías de Huatulco reef track, Oaxaca, Mexican Pacific. Zootaxa, 2422(1), 53-62.

López-Pérez, R. A., Guendulain-García, S., Granja-Fernández, R., Hernández-Urraca, V., Galván-Rowland, L., Zepeta-Vilchis, R., \& López-López, D. (2016). Reef Community Changes Associated with the 2009-2010 El Niño in the Southern Mexican Pacific. Pacific Science, 70(2), 175-190.

López-Pérez, R. A., Granja-Fernández, R., Aparicio-Cid, C., Zepeta-Vilchis, R. C., Torres-Huerta, A. M., Benítez-Villalobos, F., López-López, D. A....Valencia-Méndez, O. (2014). Corales pétreos, equinodermos y peces asociados a comunidades y arrecifes coralinos del Parque Nacional Huatulco, Pacífico sur mexicano. Revista Mexicana de Biodiversidad, 85(4), 1145-1159.

Magaña-Melgoza, P., \& Vega-González, E. (1991). Problemática ambiental del desarrollo turístico bahías de Huatulco, Oaxaca. Ingeniería Ambiental, 4, 6-12.

Mendoza, M. (2011). Clausura PROFEPA obras que afectaban el Parque Nacional Huatulco. PLANETAAZUL. Recuperado de http://www.planetaazul. com.mx/site/2011/02/02/clausura-profepa-obras-queafectaban-al-parque-nacional-huatulco

Mora, C. (2015). Perpetual struggle for conservation in a crowded world and the needed paradigm shift for easing ultimate burdens. In C. Mora (Ed.), Ecology of Fishes on Coral Reefs (pp. 289-296). Cambridge, United Kingdom: Cambridge University Press.

Moreno-Sánchez, X. G. (2009). Estructura y organización trófica de la ictiofauna del arrecife de los Frailes, $B$. C. S., México (Tesis doctoral). Instituto Politécnico Nacional, México.

Munday, P. L. (2004). Habitat loss, resource specialization, and extinction on coral reefs. Global Change Biology, 10(10), 1642-1647.

Pérez-España, H., \& Abitia-Cárdenas, L. A. (1996). Description of the digestive tract and feeding habits of the king angelfish and the Cortes angelfish. Journal of fish Biology, 48(5), 807-817.

Pielou, E. C. 1977. Mathemathical Ecology. New York: Wiley.
Piniak, G. A., \& Brown, E. K. (2008). Growth and Mortality of Coral Transplants (Pocillopora damicornis) along a Range of Sediment Influence in Maui, Hawaii. Pacific Science, 62(1), 39-55.

Pratchett, M. S., Wilson, S. K., \& Baird, A. H. (2006). Declines in the abundance of Chaetodon butterflyfishes following extensive coral depletion. Journal of Fish Biology, 69(5), 1269-1280.

Pratchett, M. S., Munday, P. L., \& Wilson, S. K. (2008). Effects of climate-induced coral bleaching on coralreef fishes. Ecological and economic consequences. Oceanography and Marine Biology: Annual Review, 46, 251-296.

PROFEPA. (2011). Clausura la PROFEPA obras que afectaban el Parque Nacional Huatulco. PROFEPA. Recuperado de: http://www.profepa.gob.mx/ innovaportal/v/3536/1/mx/clausura_la_profepa obras_que_afectaban_el_parque_nacional_huatulco. html

Ramírez-Gutiérrez, J. M. (2010). Relación del hábitat con la comunidad de peces en la bahía San Agustín, Huatulco, México (Tesis de maestría). Universidad Nacional Autónoma de México, México.

Raymundo, L. J., Couch, C. S., \& Harvell, C. D. (2008). Coral Disease Handbook: Guidelines for Assessment, Monitoring and Management. Melbourne, Australia: Currie Communications Pty Ltd.

Riegl, B. M., Heine, C., \& Branch, G. M. (1996). Function of funnel-shaped coral growth in a high-sedimentation environment. Marine Ecology Progress Series, $145,87$.

Robertson, D. R., \& Allen, G. R. (2015). Peces costeros del Pacifico Oriental Tropical: sistema de información en línea. Version 2.0. Instituto Smithsonian de Investigaciones tropicales, Balboa, República de Panamá. Recuperado de: http://www.stri.org/sftep

Rogers, C. S. (1990). Responses of coral reefs and reef organisms to sedimentation. Marine Ecology Progress Series, 62(1), 185-202.

Rogers, A., Blanchard, J. L., \& Mumby, P. J. (2014). Vulnerability of coral reef fisheries to a loss of structural complexity. Current Biology, 24(9), 1000-1005.

Tapia-García, M., García-Abad, A., Carranza-Edwards, A., \& Vázquez-Gutiérrez, F. (2007). Environmental characterization of the continental shelf of the gulf of Tehuantepec, Mexico. Geofisica Internacional, $46,249-260$.

Thomson, D. A., Findley, L. T., \& Kerstitch, A. N. (2000). Reef fishes of the Sea of Cortez: The rocky-shore fishes of the Gulf of California. Texas: University of Texas Press. 
Shannon, C. E., \& Wiener, W. (1963). The mathematical theory of comunication. Illinois: University of Illinois.

Shedrawi, G., Falter, J. L., Friedman, K. J., Lowe, R. J., Pratchett, M. S., Simpson, C. J., ... Zhang, Z. (2017). Localised hydrodynamics influence vulnerability of coral communities to environmental disturbances. Coral Reefs, 36(3), 1-12.

Simpson, E. H. (1968). Measurement of diversity. Nature, 163,688 .

Spalding, M. D., \& Jarvis, G. E. (2002). The impact of the 1998 coral mortality on reef fish communities in the Seychelles. Marine Pollution Bulletin, 44(4), 309-321.

Syms, C., \& Jones, G. P. (2000). Disturbance, habitat structure, and the dynamics of a coral-reef fish community. Ecology, 81(10), 2714-2729.
Vélez-Ascencio, O. (2009). PROFEPA sanciona con $\$ 600$ mil a Fonatur por anomalías en andador de Huatulco. La Jornada. Obtenido de http://www.jornada.unam. $\mathrm{mx} / 2009 / 02 / 14 /$ index.php? section=estados\&article $=$ $025 \mathrm{n} 2 \mathrm{es}$

Wenger, A. S., Johansen, J. L., \& Jones, G. P. (2011). Suspended sediment impairs habitat choice and chemosensory discrimination in two coral reef fishes. Coral Reefs, 30(4), 879-887.

West, J. M., \& Salm, R. V. (2003). Resistance and resilience to coral bleaching: implications for coral reef conservation and management. Conservation Biology, 17(4), 956-967.

Wilson, S. K., Graham, N. A., Pratchett, M. S., Jones, G. P., \& Polunin, N. V. (2006). Multiple disturbances and the global degradation of coral reefs: are reef fishes at risk or resilient? Global Change Biology, 12(11), 2220-2234.

See Digital Appendix at: / Ver Apéndice digital en:

revistas.ucr.ac.cr 